\title{
Inteligência Emocional no Brasil: Um Panorama da Pesquisa Científica
}

\author{
Alessandra Rodrigues Gonzaga ${ }^{1}$ \\ Janine Kieling Monteiro \\ Universidade do Vale do Rio dos Sinos
}

\begin{abstract}
RESUMO - No Brasil, estudos científicos em Inteligência Emocional (IE) são realizados apenas nas últimas duas décadas. O presente artigo é um levantamento bibliográfico a partir da pesquisa de artigos publicados em revistas científicas brasileiras, referenciados nas bases de dados INDEX PSI, LILACS, PEPSIC e SCIELO. Foram encontradas 37 publicações nacionais, sendo 12 artigos teóricos e 25 artigos empíricos. Considerando-se que já existem suficientes estudos para validação da medição de inteligência emocional, a análise conduzida remete à necessidade de mais pesquisas científicas sobre o tema no Brasil, principalmente estudos que visem avaliar a sua aplicação ou correlacionando o tema a outros construtos relevantes, em contextos variados, como educacional, social e clínico.
\end{abstract}

Palavras-chave: inteligência emocional; MSCEIT; testes de inteligência emocional.

\section{Emotional Intelligence in Brazil: An Overview from Scientific Research}

\begin{abstract}
In Brazil, scientific studies on Emotional Intelligence (EI) are being realized only during the last two decades. This article is a bibliographic survey of brazilian scientific journals cited at INDEX PSI, LILACS, PEPSIC and SCIELO. A total of 37 national publications where found: 12 theoretical articles and 25 empirical, 6 of which were qualitative studies. Seven of the 19 quantitative studies were dedicated to the validation of EI instruments, with no correlation to other constructs. The results of this survey suggest the need of complementary studies in Brazil, especially studies that assess the relation with other relevant constructs in social, educational and clinical contexts.
\end{abstract}

Key words: Emotional Intelligence, MSCEIT, tests of emotional intelligence.

O conceito de Inteligência Emocional - IE foi apresentado à comunidade científica pelos psicólogos Salovey e Mayer (1990, p. 189), em um artigo teórico, sendo definido como "a capacidade do indivíduo monitorar os sentimentos e as emoções dos outros e os seus, de discriminá-los e de utilizar essa informação para guiar o próprio pensamento e as ações". Desde então, um grande número de publicações científicas surgiu para ampliar a conceituação relacionada ao tema, modificar pressupostos existentes ou contextualizar a IE em outras interfaces psicológicas. Compreender as diferentes concepções teóricas do tema torna-se relevante para avaliar as propostas de pesquisa científica no campo da IE. Por essa razão, a introdução deste artigo se propõe a discorrer um breve histórico da conceituação da IE, do ponto de vista de seus principais teóricos.

O objetivo deste artigo foi o de fazer um levantamento de estudos científicos sobre a Inteligência Emocional publicados em revistas científicas nacionais, classificando esses estudos em artigos teóricos ou pesquisas empíricas (quantitativas e qualitativas) e destacar quais destas publicações se utilizam do modelo quadrifatorial de IE e do Mayer-Salovey-Caruso Emotional Intelligence Test (MSCEIT). Para tanto, realizou-se um levantamento bibliográfico, no período de 1996 a 2009, a partir do levantamento de artigos divulgados nas bases de dados INDEX PSI, LILACS, PEPSIC e SCIELO.

1 Endereço para correspondência: EIL - Rua Gen. Couto de Magalhães, 1155 / 501, Higienópolis - Porto Alegre, RS. CEP: 90540-030. Fone: 3307.8791.E-mail: janinekm@terra.com.br; ale.gonzaga@uol.com.br.

\section{A Inteligência Emocional}

Bar-On (2002) interpretou que o conceito inicial de IE proposto por Salovey e Mayer envolvia a autopercepção e a empatia e referia-se à capacidade de utilizar informações emocionais para guiar a cognição e o comportamento. O autor considera que o conceito de inteligência emocional origina-se das contribuições de Gardner (1994), as quais teorizavam sobre o acesso à vida emocional internalizada, feita pelo próprio indivíduo e concebiam que a classificação e diferenciação dos sentimentos em "códigos simbólicos" forneciam elementos para guiar o comportamento.

A relação entre as inteligências intrapessoal $\mathrm{e}-$ exame e conhecimento do próprio sentimento - interpessoal - capacidade de ler humores intenções e desejos dos outros (Bar-On, 2002) - é explorada por diversos pesquisadores. Para Solms (conforme citado em Taylor \& Bagby, 2000), as duas inteligências estão intimamente relacionadas, pois estando atento aos próprios sentimentos é possível estar sintonizado aos sentimentos alheios, fazendo-se uso de uma espécie de "sexto sentido".

Em uma revisão do conceito, Mayer e Salovey (1997) identificaram quatro habilidades centrais da IE: percepção das emoções, facilitação ao pensamento (integração), entendimento do conteúdo emocional e administração emocional para promover o crescimento pessoal. Nesse estudo, Mayer e Salovey não fizeram referência à inteligência interpessoal ou à habilidade e qualidade de relacionamentos, a partir do entendimento das emoções. Em outra revisão, Mayer, 
Salovey e Caruso (2002) consideraram que o termo inteligência emocional já vinha sendo empregado com muitos outros significados além do proposto pelo modelo das quatro habilidades e, repetidas vezes, vinha sendo utilizado para justificar a necessidade de uma "rebelião emocional" em direção oposta ao racionalismo vigente nas relações humanas.

As diferentes percepções do construto Inteligência Emocional não diminuem a importância das pesquisas realizadas sobre o tema. Se por um lado Goleman $(1999$, p. 39) afirma que "as capacidades implícitas da inteligência emocional são vitais para que as pessoas adquiram com êxito as competências necessárias para ter sucesso no trabalho", há pesquisadores que procuram não vincular a inteligência emocional a uma lista de qualidades desejáveis - como autoconfiança, dedicação, inovação, influência, entre outras "competências emocionais". O modelo de Goleman e também de pesquisadores que consideram capacidades não-cognitivas como formadoras da IE, ou das "competências emocionais" (Boyatzis, Goleman \& Rhee, 2002), vão de encontro ao conceito de IE como uma aptidão mental, esse último defendido por Mayer, Salovey e Caruso (2002, p. 95), que compreendem estar a IE alocada "dentro da personalidade", mais especificamente, compondo as habilidades de processamento e de entendimento das emoções.

A proposta de que o ser humano é capaz de crescer com o aprendizado e com a análise de seus conteúdos emocionais (e subjetivos) - alavancada pelo modelo de Mayer e Salovey (1997) - é bastante alinhada com a psicologia humanista de Rogers. A pessoa total é, para Rogers (1997), aquela que está aberta à suas experimentações internas e aos dados da experiência do mundo externo. Essas questões intrínsecas relacionadas à leitura pessoal dos indivíduos de seus conteúdos emocionais tornaram-se inspiração para alguns trabalhos considerados nesse artigo, em especial estudos de caso.

E qual seria a relação entre a inteligência emocional e a personalidade? Seria ela sinônimo de um determinado tipo de personalidade? Em seus estudos sobre elementos da personalidade, Mayer (1999) considera que as emoções abrangem apenas uma das quatro bases da personalidade, sendo as outras três: motivação, a cognição e a consciência, todas separadas da base emocional. Analisando o próprio modelo de personalidade, Mayer, Salovey e Caruso (2002, p. 36) compreenderam que a inteligência emocional "emprega mecanismos cognitivos e emocionais para processar aspectos emocionais do Eu, do mundo e do Eu no mundo, assim como para processar qualquer conhecimento puramente especializado da emoção". Outras propostas de pesquisa aqui consideradas acabam por relacionar a Inteligência Emocional a atributos de personalidade, algumas inclusive fazendo correlações com testes de personalidade.

\section{A escala de medição do Mayer-Salovey-Caruso Emotional Intelligence Teste (MSCEIT)}

Uma grande divisão de trabalhos relacionados à IE diz respeito ao conjunto adequado de escalas para sua medição, seja em testes já validados ou em novas propostas de medição. Em sua última revisão do tema, Bar-On (1997, p.14), caracteriza a inteligência emocional como "uma gama de aptidões, competências e habilidades não-cognitivas que influenciam a capacidade do indivíduo de lidar com as demandas e pressões do ambiente".

Em 1997, Mayer e Salovey construíram a primeira escala de Inteligência Emocional para medição do desempenho individual em tarefas de situações emocionais, por meio do teste Multifactor Emotional Intelligence Scale - MEIS. Mais recentemente, Mayer, Salovey e Caruso (2002) revisitaram o modelo das quatro habilidades de IE proposto pelo MEIS, salientando que cada uma delas pode ser separada, medida, aprendida e desenvolvida isoladamente. Os autores fizeram algumas modificações no modelo inicial e propuseram o teste MSCEIT (Mayer Salovey Caruso Emotional Intelligence Test). O construto IE avaliado pelo teste consiste na capacidade de perceber, entender e usar as emoções para facilitar o pensamento, assim como administrar as emoções para facilitar os processos cognitivos e promover o crescimento pessoal e intelectual, de uma forma bem semelhante ao modelo do teste MEIS de 1997. Nessa revisão para formar o instrumento MSCEIT, os autores passaram a considerar que o uso inteligente das emoções e seu impacto no pensamento se dão a partir dos conteúdos próprios (intrapessoais) e da leitura de emoções alheias (interpessoal).

A Tabela 1 resume as escalas do teste MSCEIT. As seções A (faces) e E (figuras) avaliam, respectivamente, a capacidade de perceber emoções em faces e ilustrações variadas. As seções B (facilitação) e F (sensações) são compostas por tarefas relacionadas à utilização da emoção para facilitação do pensamento. $\mathrm{O}$ entendimento das emoções é medido pelas tarefas das seções $\mathrm{C}$ (mudanças) e G (combinações). A administração das emoções é avaliada por meio das tarefas das seções D (gerenciamento) e H (relacionamentos). Para responder o instrumento são necessários aproximadamente 40 minutos.

Tabela 1. Escalas do Teste MSCEIT

\begin{tabular}{|c|c|c|c|}
\hline $\begin{array}{l}\text { Escala geral } \\
\text { MSCEIT }\end{array}$ & Habilidades & Tarefas & Seção \\
\hline \multirow{8}{*}{$\begin{array}{l}\text { Inteligência } \\
\text { Emocional }\end{array}$} & \multirow{2}{*}{$\begin{array}{l}\text { Percepção das } \\
\text { emoções }\end{array}$} & Faces & A \\
\hline & & Figuras & $\mathrm{E}$ \\
\hline & \multirow{2}{*}{ Uso das emoções } & Facilitação & B \\
\hline & & Sensações & $\mathrm{F}$ \\
\hline & \multirow{2}{*}{$\begin{array}{l}\text { Entendimento } \\
\text { das emoções }\end{array}$} & Mudanças & $\mathrm{C}$ \\
\hline & & Combinações & G \\
\hline & \multirow{2}{*}{$\begin{array}{l}\text { Administração } \\
\text { de emoções }\end{array}$} & Gerenciamento & $\mathrm{D}$ \\
\hline & & Relacionamentos & $\mathrm{H}$ \\
\hline
\end{tabular}

\section{Método}

Após a identificação dos estudos de IE publicados em revistas científicas nacionais, cadastrados no período de 1996 a 2009 nas bases de dados INDEX PSI, LILACS, PEPSIC e $S C I E L O$, por meio de pesquisa à Biblioteca Virtual de Psi- 
cologia (BVS), os artigos encontrados foram separados em teóricos e empíricos. Os artigos empíricos foram divididos pelo tipo de pesquisa utilizada - qualitativa ou quantitativa. De uma forma geral, foram também destacados os estudos que consideraram a metodologia quadrifatorial de IE, seja por sua aplicação ou por análise do método, utilizando-se o teste MSCEIT ou a versão anterior, o MEIS.

A base BVS também tem uma divisão de busca para teses nacionais acadêmicas (mestrado e doutorado), não contabilizados nessa pesquisa, já que muitos artigos são procedentes dos mesmos estudos dessas teses e dissertações. É o caso do artigo de Dantas e Noronha (2006), cujo grupo amostral também compôs a dissertação de Dantas (2004).

Para que a busca de artigos relacionados ao tema da Inteligência Emocional pudesse ser feita inicialmente da forma mais abrangente possível não foi feito nenhum tipo de refinamento no descritor da busca e nas bases de dados nacionais. Porém, o conjunto de 105 trabalhos identificados primeiramente entre bases de dados bibliográficas e dados de texto completo não corresponde à somatória geral de publicações de IE, já que a busca até então é redundante entre bases de dados, o que justificou a divisão das publicações e a definição de categorias de estudo.

\section{Divisões de Publicações e Categorias de Estudo}

A partir das bases de dados cobertas pela BVS foram propostas algumas divisões das publicações pesquisadas. Primeiramente, foram selecionados os artigos teóricos, ou seja, que envolveram apenas pesquisa bibliográfica, sem pesquisa aplicada. Em seguida, foram separados e classificados os artigos empíricos, em pesquisas qualitativas ou quantitativas, a partir da abordagem utilizada na análise dos dados.

Cozby (2006) compreende que existem dois métodos básicos em pesquisas para descrever e estudar o comportamento humano: qualitativo e quantitativo. A abordagem qualitativa é aquela expressa em termos não-numéricos, usando linguagem e imagens. Já a abordagem quantitativa enfatiza os dados estatísticos e utiliza os números obtidos como forma de compreender os fenômenos e variáveis estudadas.

No grupo de artigos qualitativos, foram computados aqueles relacionados a estudos de caso e pesquisa-ação. No grupo de artigos quantitativos, computaram-se estudos relacionados à validação de testes e instrumentos psicológicos e estudos correlacionais.

Considerando-se as pesquisas nacionais no campo da Inteligência Emocional divulgadas por meio das bases de dados pesquisadas no presente estudo, verificou-se que há maior concentração de estudos para mensuração de atributos de Inteligência Emocional, algumas vezes fazendo-se correlações entre variáveis de IE e outras variáveis psicossociais, de forma a verificar que tipo de influência a percepção e administração das emoções têm nas atividades sociais dos indivíduos e em suas condições psicológicas. Esses estudos quantitativos são os mais comumente encontrados em publicações científicas, havendo um pequeno número de trabalhos dedicados a considerações empíricas e revisões bibliográficas que se dediquem ao estudo da IE.
Os estudos teóricos tratam de pesquisas bibliográficas que analisam o construto de IE ou, como mais comumente encontrado, as emoções. Considerando então as linhas de pesquisa empírica, realizou-se a divisão de artigos de IE no Brasil nas seguintes categorias de estudo:

Pesquisas qualitativas, geralmente envolvendo estudo de caso de indivíduos ou grupos para análise de determinada variável psicológica, no caso da IE, envolvendo as emoções. Em alguns casos, o pesquisador busca comparar comportamentos entre dois grupos distintos, fazendo uso de análise de discurso ou comparando instrumentos psicológicos utilizados. A discussão foca-se nos desafios clínicos e terapêuticos.

Pesquisas quantitativas, envolvem investigação de indivíduos por meio de instrumentos psicológicos, com avaliação estatística dos dados. No caso do construto de Inteligência Emocional, verificou-se que são basicamente de dois tipos: (a) validações, quando o foco da pesquisa envolve a validação de alguma escala ou subescala de medição de IE. Os contextos de pesquisa são variados, geralmente estes foram desenvolvidos em espaços organizacionais ou educacionais; (b) correlações, quando é feita alguma correlação entre atributos ou habilidades de IE e outras variáveis psicológicas, havendo uso de testes correlacionais e análise estatística dos dados ou não. São exemplos de variáveis utilizadas para a comparação com a IE: bem-estar psicológico, sofrimento psíquico e stress. Estas pesquisas também são quantitativas e investigativas.

\section{Resultados e Discussão}

Os estudos teóricos envolvendo a Inteligência Emocional totalizam 13 publicações, apresentadas na Tabela 2. Esses artigos variam muito na abordagem teórica empregada, desde a psicanalítica (Souza, 2003) até discussões que abordam o âmbito da saúde pública e enfermagem (Leitão \& Arruda, 2004; Lopes Neto, 2000; Santos, Almeida \& Lemos, 1999), as quais buscam inserir as emoções no campo de estudo, mas não necessariamente o construto de inteligência emocional. No estudo de Souza (2003), a pesquisadora procurou promover uma reflexão psicanalítica sobre a importância dos aspectos emocionais no desenvolvimento da capacidade de pensar e sua contribuição na forma de solucionar problemas.

A linha teórica do teste MEIS foi explorada nos artigos de Primi (2003) e Cobêro (2003). O artigo de Cobêro analisa o livro "Manual de Inteligência Emocional" (Bar-On, 2002) e comenta os estudos do MSCEIT no contexto dos métodos de avaliação de IE. Para Primi (2003), os pesquisadores Peter Salovey, John Mayer e David Caruso foram os criadores do conceito de inteligência emocional e não o jornalista científico Daniel Goleman, popularizado por sustentar que a inteligência emocional é mais importante do que o QI. No modelo de Goleman, a inteligência emocional inclui uma série de características de personalidade, diferentemente do modelo por habilidades do MSCEIT. "É fundamental ficar claro que há uma série de pesquisas sérias com resultados importantes que contribuem para a expansão de nosso entendimento da inteligência. Essas idéias são bem diferentes da visão que acabou se popularizando por meio do livro de Goleman" (Primi, 2003, pg.72). 
Tabela 2. Artigos Teóricos que abordam IE ou emoção

\begin{tabular}{cllll}
\hline Ref & Ano & Autores & & \\
& & & & \\
\hline 1 & 2009 & Woyciekosky \& Hutz & Psicol.reflex.crit. & $\mathrm{x}$ \\
\hline 2 & 2004 & Ferreira & Conscientia & $\mathrm{x}$ \\
\hline 3 & 2004 & Leitão \& Arruda & Mundo saúde & $\mathrm{x}$ \\
\hline 4 & 2004 & Santos & Sociologias & $\mathrm{x}$ \\
\hline 5 & 2003 & Souza & Bol. Psicol. & $\mathrm{x}$ \\
\hline 6 & 2003 & Primi & Aval.psicol & $\mathrm{x}$ \\
\hline 7 & 2003 & Cobêro & PsicoUSF & $\mathrm{x}$ \\
\hline 8 & 2002 & Roberts, Flores-Mendoza \& Nascimento & Paidéia & $\mathrm{x}$ \\
\hline 9 & 2000 & Lopes Neto & Nursing SP & $\mathrm{x}$ \\
\hline 10 & 1999 & Silva Júnior, Amaral \& Cezar Neto & Rev.Bras. Psicodrama & $\mathrm{x}$ \\
\hline 11 & 1999 & Santos, Almeida. \& Lemos & Rev.Bras.Enferm & $\mathrm{x}$ \\
\hline 12 & 1997 & Correia & Estud.psicol. & Rev.Bras.Neurol. \\
\hline 13 & 1996 & Laks, Rozenthal \& Engelhardt & x & \\
\hline
\end{tabular}

$\mathrm{O}$ artigo de Roberts, Flores-Mendoza e Nascimento (2002) questiona a validade da inteligência emocional como um construto científico já que, segundo os autores, não há validade psicométrica para sustentá-la como um tipo distinto de inteligência. No entanto, esta afirmação é questionável, já que desde a década de 1990 há consenso na comunidade científica de que a inteligência emocional é um construto isolado. Em uma revisão de bibliografia científica, Salovey e Mayer (1990) analisaram estudos aparentemente não relacionados, sobre neurobiologia, medição de inteligências e Psicologia Clínica, e concluíram que todos estavam relacionados ao mesmo fenômeno: uma inteligência subjacente, que não era a mesma dos testes de Quoeficiente de Inteligência (QI), e dizia respeito às habilidades emocionais, tanto nos diálogos internos dos indivíduos como em seus relacionamentos. A expressão Inteligência Emocional foi então estabelecida, dando início a centenas de pesquisas associadas.

Considerando que esse artigo foi publicado em 2002, deve-se ponderar que desde então avanços significativos ocorreram na pesquisa de Inteligência Emocional, mesmo no Brasil. Atualmente, há uma comunidade cientifica em torno da Inteligência Emocional, que discute, utiliza e atualiza diferentes testes e modelos associados ao construto e promove congressos internacionais unicamente para apresentação de estudos científicos sobre a Inteligência Emocional. ${ }^{2}$ Para Woyciekoski e Hutz (2009), não se deve estranhar que questões específicas da pesquisa em IE ainda necessitam ser mais discutidas pela comunidade científica, o que não invalida o mérito de pesquisadores que desenvolveram

2 Como exemplo, cite-se o Consortium for Research on Emotional Intelligence in Organizations (www.eiconsortium.org), que organiza e divulga publicações científicas em torno da IE no ambiente organizacional. iniciativas sofisticadas para definir e medir o que se entende por emocionalmente inteligente - e nesse ponto os autores referiam-se especificamente aos pesquisadores Mayer e Salovey e à metodologia quadrifatorial de IE.

O artigo de Woyciekoski e Hutz (2009) destaca-se pelo aprofundado resumo das diferentes teorias envolvendo o construto da Inteligência Emocional, bem como das escalas de medição propostas nos últimos anos. Os autores concluíram que os testes mais promissores são as escalas baseadas em desempenho, as quais podem predizer alguns comportamentos importantes salientando, porém, que mesmo estas precisam ser mais bem investigadas.

Os artigos empíricos encontrados foram divididos entre pesquisas qualitativas e quantitativas, totalizando 25 estudos. A contagem total de artigos qualitativos publicados em periódicos técnico-científicos dessa amostragem é seis (Tabela 3) e o número total de artigos quantitativos é 19 (Tabela 4). Após essa seleção, alguns trabalhos qualitativos que discutem o modelo de inteligência emocional por Mayer, Salovey e Caruso (2002) foram selecionados para comentários, assim como alguns trabalhos quantitativos envolvendo o modelo quadrifatorial.

Entre as pesquisas qualitativas, destaca-se a discussão sobre o tema da inteligência emocional realizada pelos pesquisadores Neta, Garcia e Gargallo (2008), que revisaram resumos de teses e dissertações em Psicologia sobre o tema, abarcando o período de 1990 a 2006, e conduziram um estudo exploratório associado. Os autores concluíram que há um "descontínuo interesse" pelo novo construto nos cursos de Pós-Graduação de Psicologia brasileiros.

A importância da educação emocional foi tratada na pesquisa-ação de Rego, Brunelli e Rocha (2009), que analisou o tema por meio de 15 oficinas envolvendo 14 docentes, um coordenador e uma pesquisadora. A Inteligência Emocional 
Tabela 3. Pesquisas Qualitativas sobre IE

\begin{tabular}{clllll}
\hline Ref & Ano & Autores & Periódico & & \\
& & & & \\
\hline 1 & 2009 & Rego, Brunelli \& Rocha & Ensaio: Aval., Pol. Publ.Ed & $\mathrm{x}$ \\
\hline 2 & 2008 & Neta Garcia \& Gargallo & Psicol. Argum. & $\mathrm{x}$ \\
\hline 3 & 2002 & Merlo \& Barbarini & Psicol.soc. & $\mathrm{x}$ \\
\hline 4 & 2001 & Chaves & Psicol.soc. & $\mathrm{x}$ \\
\hline 5 & 2000 & Estima \& Silva & Texto \& Cont. Enferm & $\mathrm{x}$ \\
\hline 6 & 1996 & Paulo \& Perez-Ramos & Int.est.pesq.psico & $\mathrm{x}$ & \\
\hline
\end{tabular}

foi abordada nesse estudo seguindo-se a linha de estudo proposta por Goleman, Boyatzis e McKee (2002, conforme citado em Rego, Brunelli \& Rocha, 2009), sendo dividida em "competências emocionais": autoconsciência, autogestão, consciência social e administração de relacionamentos. As conclusões da pesquisa apontam que essas competências da inteligência emocional podem contribuir para a qualidade do processo de ensino-aprendizagem, conduzindo o ser humano ao equilíbrio da razão e emoção.

Dos 18 estudos quantitativos, 12 utilizaram escalas do teste MEIS (Mayer e Salovey, 1997) e do MSCEIT (Mayer Salovey \& Caruso, 2002), sendo que a totalidade desses estudos foi realizada por pesquisadores da Universidade São Francisco (SP), mais especificamente da linha de estudo de Construção, Validação e Padronização de Instrumentos de Medida, conforme Tabela 4.

Destes estudos quantitativos envolvendo o modelo quadrifatorial, cinco foram exploratórios, realizados com o objetivo de verificar a validade psicométrica da totalidade ou de parte dos testes MEIS ou MSCEIT, sem compará-lo com nenhum outro instrumento (Bueno, Santana, Zerbini \& Ramalho, 2006; Dantas \& Noronha, 2005; Muniz \& Primi, 2007; Noronha, Primi, Freitas \& Dantas, 2007; Jesus Júnior $\&$ Noronha, 2008). Os demais estudos de validade psicométrica - também da categoria de estudo "validação" na Tabela 4 - envolveram outras escalas de medição das emoções, especificamente o teste de Zulliger (Candiani, 2004) e a Medida de Inteligência Emocional - MIE (Siqueira, Barbosa \& Alves, 1999).

Alguns estudos trataram as emoções de uma forma ampla, sem considerar o construto da Inteligência Emocional (Rueda, Bartholomeu \& Sisto, 2004; Candiani, 2004; D'Avila-Bacarji, Garcia, Edna, \& Elias, 2005; Linhares et al, 2005). O estudo quantitativo de D'Avila-Bacarji, Garcia, Edna e Elias (2005) considerou o suporte parental no domínio emocional de crianças de sete a onze anos, pacientes de uma clínica de Psicologia, assim como os domínios acadêmico e desenvolvimental. As crianças foram avaliadas no nível de inteligência, desempenho escolar e problemas de comportamento. Os autores concluíram que crianças que vivem em ambientes pouco apoiadores necessitam de mais cuidados de saúde mental.
A utilização parcial de escalas do MSCEIT também foi encontrada. O estudo de Noronha, Primi, Freitas e Dantas (2007) usou apenas a metade das medições oferecidas pelo MSCEIT, mais especificamente as habilidades de Entendimento e Gerenciamento das emoções, que formam a Área Estratégica, em uma amostra de 522 trabalhadores e estudantes universitários, com o objetivo de avaliar a consistência interna dos itens do teste. Os resultados indicaram que no geral, os sub-testes apresentaram níveis de consistência interna aceitáveis considerando os padrões estabelecidos pelo Conselho Federal de Psicologia.

No estudo de Muniz, Primi e Miguel (2007), verificou-se correlações positivas entre tarefas do teste MSCEIT (Sensações e Relacionamentos) e o Inventário de Sintomas de Stress para Adultos de Lipp (ISSL), em uma amostra de 24 guardas municipais, de forma que os autores concluíram que indivíduos estressados são mais suscetíveis a experimentar emoções com menos interferência do raciocínio.

\section{Considerações Finais}

O campo de estudo pesquisado nessa revisão bibliográfica é multidisciplinar, compreendendo as áreas de Psicologia, Sociologia, Comunicação Social, Administração, Medicina, Biologia e Educação, citando-se aqui apenas as áreas mais recorrentes em pesquisas relacionadas à inteligência emocional. Para enfocar o construto da IE em pesquisas nacionais, recorreu-se às bases de dados INDEX PSI, LILACS, PEPSIC e SCIELO, por meio de consulta à BVS Psicologia, que faz a indexação dessas bases. A formatação da busca pode ter limitado o campo de pesquisa. Ainda assim, essa revisão bibliográfica pôde considerar estudos direcionados às mais variadas linhas teóricas e grupos de investigação. Considerando o conjunto de artigos encontrados nessa revisão é possível fazer algumas reflexões sobre publicações científicas em Inteligência Emocional no Brasil.

Há uma quantidade pequena de publicações de produção nacional referentes ao tema da IE, considerando-se que já existem centros de pesquisa no mundo dedicados ao assunto. Foram apenas 38, sendo a pesquisa nacional mais antiga datada de 1996, ou seja, essa quantidade é referente ao total 
Tabela 4. Pesquisas Quantitativas sobre IE

\begin{tabular}{|c|c|c|c|c|c|c|c|c|c|c|c|}
\hline Ref & Ano & Autores & Periódico & $\begin{array}{l}\text { Categoria } \\
\text { estudo }\end{array}$ & Instrumentos & Grupo amostral & $\mathbf{N}$ & 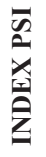 & 己 & $\frac{U}{\frac{U}{2}}$ & 号 \\
\hline 1 & 2008 & $\begin{array}{l}\text { Jesus Junior \& } \\
\text { Noronha }\end{array}$ & Psic. & Validação & MSCEIT & universitários & 191 & $\mathrm{x}$ & $\mathrm{x}$ & $\mathrm{x}$ & \\
\hline 2 & 2007 & $\begin{array}{l}\text { Muniz, Primi \& } \\
\text { Miguel }\end{array}$ & Psicol.teor.prat. & Correlação & MSCEIT e ISSL & $\begin{array}{l}\text { guardas muni- } \\
\text { cipais }\end{array}$ & 24 & $\mathrm{x}$ & $\mathrm{x}$ & $\mathrm{x}$ & \\
\hline 3 & 2007 & $\begin{array}{l}\text { Noronha, Primi, } \\
\text { Freitas, \& Dantas }\end{array}$ & Psicol. Estud & Validação & MSCEIT & $\begin{array}{l}\text { estudantes e } \\
\text { trabalhadores }\end{array}$ & 522 & $\mathrm{x}$ & & & \\
\hline 4 & 2007 & Muniz \& Primi & Aletheia & Validação & MSCEIT & $\begin{array}{l}\text { policiais mili- } \\
\text { tares }\end{array}$ & 80 & $\mathrm{x}$ & & $\mathrm{x}$ & \\
\hline 5 & 2007 & $\begin{array}{l}\text { Jesus Júnior \& } \\
\text { Noronha }\end{array}$ & Psicol.reflex.crit. & Correlação & MSCEIT, BPR-5 & universitários & 191 & & $\mathrm{x}$ & $\mathrm{x}$ & \\
\hline 6 & 2006 & $\begin{array}{l}\text { Bueno, Santana, } \\
\text { Zerbini \& Ra- } \\
\text { malho. }\end{array}$ & Psicol.teor.prat. & Validação & MSCEIT & universitários & 334 & $\mathrm{x}$ & $\mathrm{x}$ & & $\mathrm{x}$ \\
\hline 7 & 2006 & $\begin{array}{l}\text { Cobêro, Primi \& } \\
\text { Muniz }\end{array}$ & Paidéia & Correlação & MSCEIT, BPR-5, 16PF & $\begin{array}{l}\text { trabalhadores } \\
\text { empresas }\end{array}$ & 119 & $\mathrm{x}$ & $\mathrm{x}$ & & \\
\hline 8 & 2006 & $\begin{array}{l}\text { Dantas \& No- } \\
\text { ronha }\end{array}$ & Paidéia & Correlação & MSCEIT, 16 PF & universitários & 270 & $\mathrm{x}$ & & & \\
\hline 9 & 2006 & $\begin{array}{l}\text { Freitas \& No- } \\
\text { ronha }\end{array}$ & Psicol.teor.prat. & Correlação & MSCEIT, quest.próprio & $\begin{array}{c}\text { universitários } \\
\text { Psicologia }\end{array}$ & 83 & $\mathrm{x}$ & $\mathrm{x}$ & $\mathrm{x}$ & \\
\hline 10 & 2006 & $\begin{array}{l}\text { Primi, Bueno \& } \\
\text { Muniz }\end{array}$ & Psicol.cienc.prof & Correlação & MSCEIT, BPR-5, 16PF & $\begin{array}{l}\text { trabalhadores } \\
\text { empresas }\end{array}$ & 107 & $\mathrm{x}$ & $\mathrm{x}$ & & \\
\hline 11 & 2005 & Almeida & $\begin{array}{l}\text { Rev. Adm Con- } \\
\text { temp. }\end{array}$ & Correlação & Questionário próprio & $\begin{array}{c}\text { gerentes } \\
\text { portugueses }\end{array}$ & 500 & & & & $\mathrm{x}$ \\
\hline 11 & 2005 & Queroz \& Neri & Psicol.reflex. & Correlação & MIE, EDEP & $\begin{array}{l}\text { mulheres acima } \\
45 \text { anos }\end{array}$ & 120 & $\mathrm{x}$ & $\mathrm{x}$ & & $\mathrm{x}$ \\
\hline 12 & 2005 & Dantas, Noronha & Estud.pesq.psicol. & Validação & MSCEIT & universitários & 270 & $\mathrm{x}$ & $\mathrm{x}$ & $\mathrm{x}$ & \\
\hline 13 & 2005 & $\begin{array}{l}\text { D’Avila-Bacarji, } \\
\text { Garcia, Edna \& } \\
\text { Elias }\end{array}$ & Psicol.em estudo & Correlação & TDE, CBCL, EB, RAF & $\begin{array}{c}\text { crianças } 7 \text { a } 11 \\
\text { anos }\end{array}$ & 60 & & & & $\mathrm{x}$ \\
\hline 14 & 2005 & Linhares et AL & Psicol.reflex.crit. & Correlação & Raven, DFH e Escala CI & $\begin{array}{c}\text { crianças } \\
8 \text { a } 10 \text { anos }\end{array}$ & 40 & & & & $\mathrm{x}$ \\
\hline 15 & 2004 & $\begin{array}{l}\text { Rueda, Bartholo- } \\
\text { meu \& Almeida }\end{array}$ & Psicol.Argum & Correlação & $\begin{array}{c}\text { Teste de Bender, Dese- } \\
\text { nho FH }\end{array}$ & crianças & 312 & $\mathrm{x}$ & & & \\
\hline 16 & 2004 & Candiani & Psic. & Validação & Teste de Zulliger & $\begin{array}{c}\text { universitários } \\
\text { Psicologia }\end{array}$ & 50 & & & & $\mathrm{x}$ \\
\hline 17 & 2003 & Bueno \& Primi & Psicol.reflex.crítica & Correlação & $\begin{array}{c}\text { Subescala MEIS, } 16 \mathrm{PF}, \\
\text { BPR-5 }\end{array}$ & $\begin{array}{l}\text { universitários } \\
\text { Psicologia }\end{array}$ & 76 & $\mathrm{x}$ & $\mathrm{x}$ & & $\mathrm{x}$ \\
\hline 18 & 1999 & $\begin{array}{l}\text { Siqueira, Barbosa } \\
\& \text { Alves }\end{array}$ & Psicol.teor.prat. & Validação & MIE & aleatória & 972 & $\mathrm{x}$ & & & \\
\hline
\end{tabular}

Legenda dos instrumentos: MEIS - Multifactor Emotional Intelligence Scale; MSCEIT - Mayer, Salovey e Caruso Emotional Intelligence Test; ISSL Inventário de Sintomas de Stress para Adultos de Lipp ; 16PF - Inventário de 16 Fatores de Personalidade; EDEP - Escala de Desenvolvimento Pessoal, BPR-5 - Bateria de Provas de Raciocínio; DSF - Desenho da Figura Humana, Escala CI - Escala Comportamental Infantil. 
de estudos realizados nos últimos 13 anos, incluindo-se 13 artigos teóricos encontrados;

Os estudos teóricos em IE têm enfoques bastante variados, mas é possível perceber o aprimoramento dos artigos. Dos 13 artigos teóricos encontrados, apenas três consideravam a inteligência emocional como um construto psicológico (Woyciekoski \& Hutz, 2009; Cobêro, 2003; Primi, 2003), sendo que o restante discute o assunto de forma mais abrangente, sem necessariamente falar da inteligência emocional. Há um artigo questionando a IE como construto psicológico, sustentando que não há validade psicométrica para que possamos considerá-la um tipo de inteligência (Roberts, Flores-Mendoza \& Nascimento, 2002). O artigo de Woyciekoski e Hutz (2009) representa, no entanto, a chegada em um novo patamar de entendimento do construto IE no Brasil, já que resume boa parte das indagações atuais da comunidade científica internacional em relação ao tema;

Muitos artigos não necessariamente tratam do construto psicológico "inteligência emocional", mas sim consideram as emoções humanas de uma forma mais ampla em suas investigações. Entre os seis artigos qualitativos encontrados, a única exceção é o estudo de Neta, Garcia e Gargallo (2008), que faz uma apreciação do construto de IE em estudos brasileiros. Nos estudos quantitativos, com exceção dos artigos que utilizaram escalas ou subescalas do teste MEIS, do teste MSCEIT ou a escala da Medida de Inteligência Emocional (MIE), os demais compreendem as emoções em seus grupos amostrais tendo por base outras linhas teóricas, sem considerar o construto IE (Rueda, Bartholomeu \& Sisto, 2004; Candiani, 2004, Linhares et al, 2005; D’Avila-Bacarji, Garcia, Edna, \& Elias, 2005);

Grande parte da pesquisa quantitativa de IE no Brasil é dedicada a validar ou aplicar testes de IE ou subescalas associadas. Dos 19 estudos quantitativos, sete foram para validação de escalas de medição de inteligência emocional, sem correlação com outros construtos, o que sugere que estes estudos ainda não avançaram na aplicação do conceito;

$\mathrm{O}$ assunto da IE já é reconhecido internacionalmente. $\mathrm{O}$ teste MSCEIT, dos pesquisadores Mayer, Salovey e Caruso já foi aplicado em centenas de pesquisas no mundo inteiro e a última revisão do instrumento é de 2002. No Brasil, as escalas do MSCEIT já foram discutidas em dois artigos teóricos (Woyciekoski \& Hutz, 2009; Primi, 2003), sendo que foram encontradas evidências de validade para o instrumento MEIS (Bueno \& Primi, 2003) e realizados estudos psicométricos também com o MSCEIT (Freitas \& Noronha, 2006; Noronha \& Primi, 2007). Um dos objetivos desse artigo foi o de examinar a utilização do teste MSCEIT em artigos publicados no Brasil. Verificou-se que das 18 pesquisas quantitativas encontradas, 11 utilizaram o teste MSCEIT, atestando a preferência nesse instrumento para medição de inteligência emocional entre os testes escolhidos por pesquisadores nacionais. Entre as vantagens de uso do MSCEIT estão sua aplicação em diferentes públicos, já realizada em estudos nos cinco continentes, assim como sua escala de medição por habilidades, que diferencia-se das demais escalas de auto-avaliação. No entanto, estudos complementares e a validação da versão MSCEIT para o Brasil, incluindo tradução e adaptações pertinentes, ainda são necessários, de forma a avançar no conhecimento e na condução de estudos correlacionais, disseminando os estudos em IE no Brasil;

O passo seguinte ao processo de mensuração de um conceito é seu desenvolvimento aplicado. Há alguns anos, já existem estudos de desenvolvimento da IE na forma de intervenções clínicas ou estudos experimentais em empresas (Jennings \& Palmer, 2007; Law, Wong, Huang \& Li, 2008; Nelis, Quoidback, Mikolajczak \& Hansenne, 2009). A pesquisa científica no Brasil ainda não chegou neste estágio.

A análise aqui conduzida remete à necessidade da realização de mais pesquisas científicas sobre o tema no Brasil, tanto quantitativas quanto qualitativas, principalmente visando a aplicação da Inteligência Emocional em contextos organizacionais, educacionais e sociais, dada a importância do entendimento e utilização positiva das emoções nas relações inter e intrapessoais dos indivíduos.

\section{Referências}

Almeida, M. C. (Ed.). (2005). Conjunto de guias sobre serviços e politicas de saúde mental. Barcelona: Edimsa.

Bar-On, R. (1997). Bar On emotional quotient inventory (EQ-i): Technical manual. Toronto: Multi-Health Systems.

Bar-On, R. (2002). Inteligência social e emocional: Visões do Emotional Quotient Inventory. In R. Bar-On, \& J. D. A. Parker (Eds.), Manual de inteligência emocional (pp. 266-283). Porto Alegre: Artmed.

Boyatzis, R., Goleman, D., \& Rhee, K. (2002). Agrupando as competências da inteligência emocional: Visões do Emotional Competence Inventory. In R. Bar-On \& J. D. A. Parker (Eds.), Manual de inteligência emocional (pp. 252-265). Porto Alegre: Artmed.

Bueno, J. M. H., \& Primi, R. (2003). Inteligência emocional: um estudo de validade sobre a capacidade de perceber emoções. Psicologia Reflexão e Crítica, 16(2), 279-29.

Bueno, J. M. H, Santana, P. R., Zerbini, J., \& Ramalho, T. B. (2006). Inteligência emocional em estudantes universitários. Psicologia: Teoria e Pesquisa, 22(3), 305-316.

Candiani, D. A. (2004). Precisão do Teste de Zulliger. Psic: revista da Vetor Editora, 5(1), 16-21.

Chaves, J. C.(2001). Estratégias de apropriação das emoções no ambiente de trabalho. Psicologia e Sociedade, 13(1), 46-63.

Cobêro, C. (2003). Manual de inteligência Emocional. Psico-USF, 8 (1), 95-96.

Cobêro, C., Primi, R., \& Muniz, M. (2006). Inteligência emocional e desempenho no trabalho: um estudo com MSCEIT, BPR-5 e 16PF. Paidéia, 16(35), 337-348.

Correia, M. F. B. (1997). Inteligência emocional: da revolução à controvérsia. Estudos em Psicologia, 2(2), 413-419.

Cozby, P. (2006). Métodos de pesquisa em ciências do comportamento. São Paulo: Editora Atlas.

Dantas, M. A. (2004). Inteligência emocional: Validade discriminativa entre MSCEIT e 16 PF em universitários (Dissertação de mestrado não publicada). Universidade de São Francisco, São Paulo.

Dantas, M. A., \& Noronha, A.P. (2005). Inteligência emocional: Parâmetros psicométricos de um instrumento de medida. Estudos e Pesquisas em Psicologia, 5(1), 59-72. 
Dantas, M. A., \& Noronha, A. P. (2006). Validade discriminante entre MSCEIT e 16 PF. Paidéia, 16(33), 59-70.

D’Avila-Bacarji, K. M., Garcia, M., Edna, M., \& Elias, L. C. S. (2005). Suporte parental: um estudo sobre crianças com queixas escolares. Psicologia em Estudo, 10(1), 107-115.

Estima, S. L., \& Silva, A. L. (2000). O cuidado no desenvolvimento de capacidades intra/interpessoais de trabalhadoras de enfermagem. Texto \& Contexto Enfermagem, 9(2), 348-361.

Ferreira, R. (2004). Inteligência parapsíquica e invéxis. Conscientia, 8(3), 175-179.

Freitas, F. A., Noronha, A. P. (2006). Inteligência emocional e avaliação de alunos e supervisores: evidências de validade. Psicologia: Teoria e Prática, 8(1), 77-93.

Gardner, H. (1994). Estruturas da mente (Sandra Costa, Trad.). Porto Alegre: Artmed. (Obra original publicada em 1983).

Goleman, D. (1999). Trabalhando com a inteligência emocional. Rio de Janeiro: Objetiva.

Jesus Junior, A. G., \& Noronha, A. P. (2008). Parâmetros psicométricos do Mayer Salovey Caruso Emotional Intelligence Test: MSCEIT. Psic: revista da Vetor Editora, 9(2), 145-153.

Jesus Junior, A. G., \& Noronha, A. P.(2007). Inteligência emocional e provas de raciocínio: um estudo correlacional. Psicologia, Reflexão e Crítica, 20(3), 480-489.

Laks, J., Rozenthal, M., \& Engelhardt, E. (1996, set-out). Neuropsicologia: X - Neuropsicologia das emoções. Revista Brasileira de Neurologia, 32(5), 177-81.

Law, K. S., Wong, C. S., Huang, G. H., \& Li, X. (2008). The effects of emotional intelligence on job performance and life satisfaction for the research and development scientists in China. Asia Pacific Journal of Management, 25, 51-69.

Leitão, I. M. \& Arruda, R. B. (2004). Clima organizacional: novos rumos no perfil do enfermeiro e desafios a assistência de enfermagem. Mundo Saúde, 28(2), 151-159.

Linhares, M. B. M., Chimello, J. T., Bordin, M. B. M., Carvalho, A. E. V. \& Martinez, F. E. (2005). Desenvolvimento psicológico na fase escolar de crianças nascidas pré-termo em comparação com crianças nascidas a termo. Psicologia, Reflexão e Crítica, 18(1), 109-117.

Lopes Neto, D. (2000). Liderança transformacional: A arte de administrar com inteligência emocional. Nursing, 3(21), 16-19.

Mayer, J. D. (1999). Emotional intelligence: Popular or scientific psychology? APA Monitor, 30(8), 50.

Mayer, J., \& Salovey, P. (1997). What is emotional intelligence? New York: Basic Books.

Mayer, J., Salovey, P., \& Caruso, D. (2002). Selecionando uma medida para a inteligência emocional: Em defesa das escalas de aptidão. In R. Bar-On \& J. Parker (Eds.), Manual de inteligência emocional (pp. 237-251). Porto Alegre: Artmed.

Merlo, A. R. C., \& Barbarini, N. (2002). Reestruturação produtiva no setor bancário brasileiro e sofrimento dos caixas executivos: um estudo de caso. Psicologia \& Sociedade, 14(1), 103-122.

Muniz, M., \& Primi, R. (2007). Inteligência emocional e desempenho em policiais militares: Validade de critério do MSCEIT. Revista Aletheia, 25, 66-81.

Muniz, M., Primi, R., \& Miguel, F. K. (2007). Investigação da inteligência emocional como fator de controle do stress em guardas municipais. Psicologia: Teoria e Prática, 9(1), 27-41.

Neta, N. F. A., Garcia, E., \& Gargallo, I. S. (2008). A inteligência emocional no âmbito acadêmico: Uma aproximação teórica e empírica. Psicologia Argumento, 26 (52), 11-22.
Noronha, A. P. P., Primi, R., Freitas, F. A., \& Dantas, M. A. (2007). Análise dos itens do Mayer-Salovey-Caruso Emotional Intelligence Test: Escalas da área estratégica. Revista Psicologia em Estudo, 12(2), 415-422.

Paulo, M. S. L, Perez-Ramos, A. M. Q. (1996). Distúrbios emocionais em criancas com dificuldades de aprendizagem escolar. Interações: Estudo e Pesquisa em Psicologia, 1(2), 127-138.

Primi, R. (2003). Inteligência: Avanços dos modelos teóricos e nos instrumentos de medida. Avaliação Psicológica, 1(2), 67-77.

Primi, R., Bueno, J. M. H., \& Muniz, M. (2006). Inteligência emocional: Validade convergente e discriminante do MSCEIT com a BPR-5 e o 16 PF. Psicologia Ciência e Profissão, 26(1), 26-45.

Queroz, N.C.\& Neri, A.L.. (2005). Bem-estar psicológico e inteligência emocional entre homens e mulheres na meia-idade e na velhice. Psicologia, Reflexão e Crítica, 18 (2), 292-299.

Rêgo, C., Brunelli, C. A., \& Rocha, N. M. (2009). Avaliando a educação emocional: subsídios para um repensar da sala de aula. Ensaio: Avaliação e Políticas Públicas em Educação, 17(62), 135-152

Roberts, R. D., Flores-Mendoza, C. E., \& Nascimento, E. (2002). Inteligência emocional: Um construto científico? Paidéia, 12(23), 77-92.

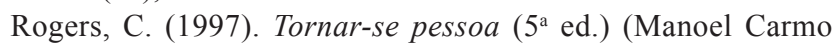
Ferreira, Trad.). São Paulo: Martins Fontes. (Obra original publicado em 1961).

Rueda, F.J.M., Bartholomeu, D. S., Sisto, F. (2004). Desenho da figura humana e teste de Bender: um estudo correlacional. Psicologia Argumento, 22 (39), 39-44.

Salovey, P., \& Mayer, J. D. (1990). Emotional Intelligence. Imagination, Cognition and Personality, 9, 185-211.

Santos, L. M.; Almeida, F.L. \& Lemos, S., C. (1999). Inteligência emocional: testando a enfermagem do futuro. Revista Brasileira de Enfermagem, 52 (3), 401-12.

Santos, M. J. N. (2004). Gestão de recursos humanos: teorias e práticas. Sociologias, 12, 142-158.

Silva Júnior, A., Amaral, G. B., \& Cezar Neto, H. C. (1999). Psicodrama e inteligência emocional. Revista Brasileira de Psicodrama, 7(1), 107-112.

Siqueira, M. M., Barbosa, N. C., \& Alves, M. T. (1999). Construção e validação fatorial de uma medida de inteligência emocional. Psicologia: Teoria e Pesquisa, 15(2), 143-152.

Souza, A. S. L. (2003). O pensamento como aquisição do desenvolvimento psíquico: reflexões sobre os aspectos emocionais da inteligência. Boletim de Psicologia, 53(118), $1-12$.

Taylor, G., \& Bagby, M. (2000). Uma visão geral do constructo de alexitimia. In R. Bar-On \& J. Parker (Eds.), Manual de inteligência emocional (pp. 47-64). Porto Alegre: Artmed.

Woyciekosky, C. \& Hutz, C. S. (2009). Inteligência emocional: teoria, pesquisa, medida, aplicações e controvérsias. Psicologia, Reflexão e Crítica, 22 (1), 1-11.

Recebido em 15.09.2009

Primeira decisão editorial em 26.01.2010

Versão final em 22.02.2010

Aceito em 21.06.2010 